\title{
Personality and Cognitive Processing of Affective Information
}

\author{
Cheryl L. Rusting \\ Randy J. Larsen \\ University of Michigan
}

Several studies have demonstrated that the personality traits of extraversion and neuroticism are positively correlated with susceptibilities to positive and negative affect, respectively. These findings are often explained in terms of Jeffrey Gray's theory of personality, which predicts that extraverts and neurotics are differentially susceptible to stimuli that generate positive and negative emotional states. The current research provides a further test of Gray's theory using a series of cognitive tasks with positive and negative stimuli. In Study 1, participants completed a word-fragment completion task, a reaction-time task, and a recall task. Results showed that extraversion was generally related to performance when stimuli were positive but not when stimuli were negative or neutral. Study 2 replicated these findings and demonstrated that the relationships between personality and performance were not mediated by current mood state. Discussion focuses on integrating a cognitive analysis of personality with existing biological theories.

$\mathbf{U}$

nderstanding relationships between personality and emotion has been the subject of a considerable amount of research in recent years. Some researchers have suggested that certain personality traits can be reliably mapped onto different kinds of emotional experiences (Larsen \& Diener, 1992; Meyer \& Shack, 1989; Rusting \& Larsen, 1995). In particular, the personality dimensions of extraversion and neuroticism tend to correlate with the mood factors of positive and negative affect. Many studies have consistently demonstrated positive relationships between extraversion and positive affect and between neuroticism and negative affect (Costa \& McCrae, 1980; Emmons \& Diener, 1985; Larsen \& Ketelaar, 1989, 1991; Meyer \& Shack, 1989; O'Malley \& Gillette, 1984; Rusting \& Larsen, 1995; Watson \& Clark, 1992).

These findings are often interpreted as evidence for temperamental differences in the predisposition to ex- perience positive or negative affect. However, the majority of studies examining the relationships between these personality traits and positive and negative mood experiences have used primarily self-report questionnaire measures of personality and then correlated them with self-report questionnaire measures of affect. Because of the correlational nature of these studies, they have not directly tested the proposition that extraversion and neuroticism predispose individuals to experience positive and negative affect.

Larsen and Ketelaar $(1989,1991)$ attempted to provide some direct evidence for the predisposition of extraverts and neurotics to experience positive and negative affect. Conceptualizing positive and negative affect as the "subjectively reportable representations" of sensitivity to reward and punishment, Larsen and Ketelaar (1989, p. 1222, 1991) hypothesized that extraversion and neuroticism should be related to susceptibility to positive and negative affective stimuli, respectively. The authors used reactivity to positive and negative mood induction procedures as measures of sensitivity to positive and negative stimuli. They found that extraverts showed greater emotional responsivity to positive mood inductions than did introverts and that individuals high in

Authors' Note: During the preparation of this article, Cheryl L. Rusting was supported by a graduate fellowship from the National Science Foundation. An earlier version of this article was presented at the May 1995 Midwestern Psychological Association conference, Chicago. We thank Kevin Bennett, Karen Lutwin, and Michael Wood for their assistance in data collection. We also express our gratitude to Oliver John and three anonymous reviewers for their detailed and very helpful comments on an earlier version of this article. Address correspondence to Cheryl L. Rusting, now at the Department of Psychology, State University of New York at Buffalo, Buffalo, NY 14260-4110, e-mail crusting@acsu.buffalo.edu.

PSPB, Vol. 24 No. 2, February 1998 200-213

(C) 1998 by the Society for Personality and Social Psychology, Inc. 
neuroticism were more emotionally responsive to negative mood inductions than were stable individuals. In other words, extraversion was the best predictor of positive affective reactions, and neuroticism was the best predictor of negative affective reactions when individuals were exposed to the same emotional stimulus (i.e., mood induction).

Larsen and Ketelaar $(1989,1991)$ interpreted their findings based on Gray's (1981) theory of personality. According to Gray's theory, two emotion-based brain systems regulate behavior in the presence of rewards and punishments. The behavioral activation system (BAS) is sensitive to cues signaling reward or the removal of expected punishment, and the behavioral inhibition system (BIS) is sensitive to cues signaling punishment or frustrative nonreward. Each of these systems is thought to produce a personality dimension such that heightened sensitivity to reward is indicative of extraversion, whereas heightened sensitivity to punishment is linked to neuroticism. Larsen and Ketelaar suggested that extraversion and neuroticism can be thought of as rooted in these biologically based systems, which directly promote differential responsivity to positive and negative stimuli.

The purpose of the present series of studies was to extend this research into the cognitive domain. If extraversion and neuroticism predispose individuals to respond to positive and negative emotional stimuli, respectively, then their effects should also be apparent in the cognitive processing of affective stimuli. For example, the predisposition for extraverts to be sensitive to reward could be accompanied by a cognitive elaboration of positive, reward-relevant information in memory, making extraverts better at processing signals of reward. Similarly, the predisposition for individuals high in neuroticism to be sensitive to punishment could be accompanied by cognitive elaboration of negative, punishment-relevant information in memory, making neurotics better at processing signals of punishment.

Such cognitive elaboration is consistent with Bower's $(1981,1987)$ network theory of affect, which has been a popular model in the emotion and cognition literature. According to this theory, emotions impose a fundamental organizational structure on information in memory. Each specific emotion, such as sadness, anger, joy, or fear, is represented by a particular emotion node within a cognitive network consisting of emotion-related memories and cognitions that have been previously encoded and organized in memory. When a particular node is activated, this activation is channeled through its network of connections to evoke emotion-related memories and cognitions. Although Bower's (1981) model was originally formulated to account for the effects of mood states on cognitive processing, several theorists have hinted that the model could be easily extended to account for the effects of stable personality traits (e.g., Clark \& Teasdale, 1985; Mayer \& Volanth, 1985; Teasdale \& Russell, 1983). Individuals could differentially develop well-elaborated associative networks surrounding particular emotion nodes due to their heightened predispositions to those emotions.

This type of model can be applied to the personality traits of extraversion and neuroticism. Positive and negative emotion nodes should include emotional content related to positive and negative information in memory. The associative network for an individual high in neuroticism would have many interconnections among these negative emotional concepts, events, and images associated with punishment. Similarly, the associative network for an extravert would have many interconnections among positive emotional concepts, events, and images associated with reward. Hedonic information consistent with the content of these networks should attract attention and be easily brought to mind because of the multiple pathways and strong interconnections. Extraverts and neurotics should, therefore, be especially quick at noticing, and better at remembering, positive and negative emotional material, respectively.

A few studies have cited evidence for the selective processing of positive and negative emotional information associated with these personality traits. Most of this research, however, has investigated trait-congruent memory only. For example, following the presentation of a list of positive, negative, and neutral words, highneuroticism individuals recall more negative words than do low-neuroticism individuals (Bradley \& Mogg, 1994; Bradley, Mogg, Galbraith, \& Perrett, 1993; Desrosiers \& Robinson, 1992; Martin, Ward, \& Clark, 1983; Young \& Martin, 1981). Individuals high in neuroticism also retrieve negative personal memories faster (Lishman, 1974; Okun, Stock, Snead, \& Wiermaa, 1987) and more frequently (Mayo, 1983; Ruiz-Caballero \& Gonzalez, 1995) than do individuals low in neuroticism. Mayo (1983) reported that neuroticism accounted for the most variance in a regression analysis (which also included mood state, anxiety, and extraversion as predictors) in retrieval of unpleasant memories. Extraverts, on the other hand, tend to retrieve pleasant memories (Mayo, 1983, 1989).

Although the evidence for extraverts' and neurotics' selective memory for positive and negative emotional material appears reliable, very little research has examined the relationships between extraversion and neuroticism and selective processing in other cognitive domains. It is necessary to investigate other cognitive processes because the evidence for emotion-congruent processing is not always uniform across different cognitive domains (e.g., Williams, Watts, McLeod, \& Mathews, 
1988). According to the network model described above, elaboration of positive and negative emotional concepts in memory for extraverts and neurotics should also lead to faster processing of positive and negative emotional stimuli and an increased likelihood that positive and negative emotional concepts will be brought to mind.

In the current series of studies, we assessed extraverts' and neurotics' differential processing of positive and negative emotional stimuli using a variety of cognitive tasks (reaction time, word-fragment completion, and free recall). These tasks allowed us to measure speed of response and accessibility of positive and negative emotional material, in addition to memory retrieval. By using a series of different cognitive tasks, we hoped to extend previous research on extraversion and neuroticism and cognitive processing of affective information.

To assess speed of responding, participants completed a reaction-time task, in which they were presented with a stimulus and asked to determine its valence as quickly as possible. If extraverts have more elaborate cognitive networks with stronger links among positive emotional concepts in memory, then they should be faster in responding to positive stimuli, because positive associations should quickly come to mind for these individuals. Likewise, if neurotics have more elaborate cognitive networks with stronger links among negative emotional concepts in memory, then they should be faster in responding to negative stimuli, because negative associations should quickly come to mind for these individuals. According to this reasoning, extraversion should relate to faster reaction times for positive stimuli but not for negative or neutral stimuli. Neuroticism, on the other hand, should relate to faster reaction times for negative stimuli but not for positive or neutral stimuli.

According to a cognitive network interpretation, extraverts should also be especially likely to impose positive or negative interpretations onto ambiguous emotional concepts. To test this idea, we presented participants with a series of ambiguous word fragments, half of which could be completed as positive or neutral and half of which could be completed as negative or neutral. Extraversion should relate to the completion of positive meanings of words (because these positive meanings should be more accessible in memory) but not to the completion of negative meanings. Neuroticism should relate to the completion of negative meanings of words but not to the completion of positive meanings.

Our predictions for the free-recall task followed similar reasoning. If positive emotional material is more elaborated in memory for extraverts and negative emotional material is more elaborated in memory for neurotics, then these traits should differentially relate to the recall of positive and negative affective stimuli. Extraversion should relate to better recall of positive words but not negative or neutral words. Neuroticism, on the other hand, should relate to better recall of negative words but not positive or neutral words.

The advantages of the present research over prior studies of personality and emotion are twofold. First, the current series of studies uses performance measures, rather than traditional self-report methods, to test the proposition that extraversion and neuroticism are characterized by differential sensitivities to positive and negative affective stimuli. Studies using self-reports of personality and emotion are subject to self-presentational biases, especially if participants are aware of the focus of the study on emotion. Prior studies of personality and emotion have often relied on self-report measures of emotion (e.g., Costa \& McCrae, 1980). Even the Larsen and Ketelaar $(1989,1991)$ studies used self-report measures to assess affect intensity following the induction of an emotional state. By using performance measures, the present research avoids problems associated with possible self-presentational biases. The focus of the study on emotion is not immediately obvious, and participants have less control over their responses on the cognitive tasks than they do on a self-report measure of affect.

Second, the current series of studies uses a variety of different tasks to assess relationships between personality and sensitivity to emotional information across several cognitive domains. Most of the prior literature investigating relationships between extraversion and neuroticism and cognitive processing has focused on only one domain (memory retrieval). The present research uses multiple cognitive methods to assess differential sensitivity to positive and negative affective stimuli.

\section{STUDY 1}

Method

Participants. A total of 122 right-handed introductory psychology students (60 men, 62 women) participated in the study for partial course credit.

Procedure. Participants were run individually, in approximately 50-min sessions. Upon arrival at the laboratory, each participant listened to an overview of the procedure, signed an informed-consent form, and completed the Extraversion and Neuroticism scales of the Eysenck Personality Questionnaire (EPQ) (Eysenck \& Eysenck, 1972). The cover story presented the research simply as a study of the performance of college students on cognitive tasks.

After completing the EPQ participants were given the word-fragment completion task in which they were instructed to fill in the missing letters in each item to complete a word. The task consisted of 40 ambiguous stimuli. Of the items, 20 could be completed as either 
positive or neutral (e.g., E_A_E D could be completed as elated or erased), and 20 could be completed as either negative or neutral (e.g., A N G _ _ could be completed as anger or angle). To eliminate possible ceiling effects, only those items that were completed using between $20 \%$ and $80 \%$ of both answers (positive and neutral, or negative and neutral) were included in the analyses. Eight items were eliminated because more than $80 \%$ or fewer than $20 \%$ of the participants chose one of the answers. The 32 remaining items and some possible answers are listed in Appendix A.

Next, participants were asked to sit in front of a computer screen at an adjacent desk. The reaction-time task was introduced as a measure of "speed of responding." Participants were told that they would see a word or picture appear on the screen and they would need to decide whether it was positive, negative, or neutral. The presentation of each stimulus was preceded by a "+" prompt and a preparatory tone. Each stimulus appeared in reverse on a white background, to reduce possible image aftereffects. Participants were instructed to decide whether the stimulus was positive, negative, or neutral and to press the corresponding left, right, or up-arrow key with their right-hand index finger (positive, negative, and neutral overlays were placed over the keys). Between responses, participants were told to rest their index fingers on the bottom-arrow key. The order of positive and negative response keys was counterbalanced, so that half of the participants were instructed to press the left-arrow key if the stimulus was negative and the rightarrow key if the stimulus was positive. The other half pressed the right-arrow key if the stimulus was negative and the left-arrow key if the stimulus was positive. Participants were told to respond as quickly as possible but to avoid making errors by pressing the wrong key or responding before the stimulus appeared on the screen.

Prior to beginning the reaction-time task, each participant completed two blocks of practice trials, each consisting of 20 items similar to the ones presented in the real reaction-time task. For the real task, each participant completed four blocks of trials, consisting of 60 items each (20 positive, 20 negative, and 20 neutral). Two of the blocks consisted of words (see Appendix B for a list of the items), and two consisted of line drawings of faces. ${ }^{1}$ Half of the participants received the word-item blocks first, and half received the face-item blocks first. The order of presentation of the items in each block was randomized prior to each run. The word block and face block were each administered twice, yielding a total of 40 positive, 40 negative, and 40 neutral words and faces administered to each participant.

Following the last reaction-time trial, participants were asked to recall as many words as they could from the reaction-time task. They were given $4 \mathrm{~min}$ to recall
TABLE 1: Descriptive Statistics for Cognitive Tasks, Study 1

\begin{tabular}{lcc}
\hline & $\mathrm{M}$ & $\mathrm{SD}$ \\
\hline Word-fragment completion & & \\
$\quad$ Positive words & 12.72 & 2.41 \\
$\quad$ Negative words & 12.39 & 2.75 \\
Verbal reaction time & & \\
$\quad$ Positive words & 702.24 & 78.77 \\
$\quad$ Negative words & 714.52 & 74.84 \\
$\quad$ Neutral words & 699.51 & 72.39 \\
Nonverbal reaction time & & \\
Positive faces & 564.29 & 60.70 \\
Negative faces & 572.96 & 66.47 \\
$\quad$ Neutral faces & 552.20 & 66.72 \\
Verbal reaction-time errors & & \\
$\quad$ Positive words & 3.80 & 3.10 \\
Negative words & 3.38 & 2.71 \\
$\quad$ Neutral words & 3.91 & 4.47 \\
Nonverbal reaction-time errors & & \\
Positive faces & 1.91 & 2.63 \\
Negative faces & 2.31 & 6.26 \\
$\quad$ Neutral faces & 2.18 & 3.74 \\
Free recall & & \\
Positive words & & \\
Negative words & 5.57 & 1.69 \\
Neutral words & 4.93 & 1.82 \\
\hline
\end{tabular}

and record the words onto a blank sheet of paper. After completing the recall task, each participant was thoroughly debriefed and thanked for participating.

Results

\section{WORDFRAGMENT COMPLETION}

Overall performance. Means and standard deviations for the number of items participants completed as positive or negative are presented in the top portion of Table 1 . A repeated measures ANOVA indicated that participants completed relatively equal numbers of words as positive or negative, $F(1,121)=1.60, n s$.

Personality and differential performance. To test whether extraverts completed more words as positive than did introverts and whether individuals high in neuroticism completed more words as negative than did stable individuals, we computed correlations between extraversion and neuroticism and positive and negative words completed. ${ }^{2}$ If extraverts are especially sensitive to positive information, then there should be a positive relationship between extraversion and the number of words completed as positive but not with the number of words completed as negative. Likewise, if neurotics are especially sensitive to negative information, then there should be a positive relationship between neuroticism and the number of words completed as negative but not with the number of words completed as positive. These correlations are presented in the top portion of Table 2. Extraversion did not significantly correlate with any of 
TABLE 2: Correlations Between Personality and Performance on Cognitive Tasks, Study 1

\begin{tabular}{|c|c|c|c|}
\hline & $\begin{array}{c}\text { Extraversion } \\
\mathrm{r}\end{array}$ & $\begin{array}{c}\text { Neuroticism } \\
\mathbf{r}\end{array}$ & $\begin{array}{c}\text { Difference } \\
\mathrm{t}\end{array}$ \\
\hline \multicolumn{4}{|c|}{ Word-fragment completion } \\
\hline Positive words & .09 & .14 & -0.53 \\
\hline Negative words & .05 & $.18^{*}$ & 1.37 \\
\hline \multicolumn{4}{|c|}{ Verbal reaction time } \\
\hline Positive words & $-.21 *$ & -.05 & $1.90^{*}$ \\
\hline Negative words & -.07 & -.08 & 0.10 \\
\hline Neutral words & -.09 & -.06 & -0.31 \\
\hline \multicolumn{4}{|c|}{ Nonverbal reaction time } \\
\hline Positive faces & -.14 & .01 & -1.57 \\
\hline Negative faces & -.12 & -.09 & -0.31 \\
\hline Neutral faces & -.10 & -.01 & -0.94 \\
\hline \multicolumn{4}{|c|}{ Verbal reaction-time errors } \\
\hline Positive words & $-.23 * *$ & -.01 & $-2.34^{*}$ \\
\hline Negative words & -.16 & $-.19 *$ & 0.32 \\
\hline Neutral words & .03 & -.03 & 0.62 \\
\hline \multicolumn{4}{|c|}{ Nonverbal reaction-time errors } \\
\hline Positive faces & $-.24 * *$ & -.12 & -1.29 \\
\hline Negative faces & -.01 & -.07 & 0.62 \\
\hline Neutral faces & -.10 & -.03 & -0.73 \\
\hline \multicolumn{4}{|l|}{ Free recall } \\
\hline Positive words & $.20^{*}$ & .00 & $2.11^{*}$ \\
\hline Negative words & .06 & .13 & -0.73 \\
\hline Neutral words & -.09 & -.05 & -0.42 \\
\hline
\end{tabular}

$* p<.05 . * * p<.01$.

the word-completion variables. Neuroticism did, however, significantly correlate with the number of negative words completed and not with the number of positive words completed. The results, therefore, supported only our prediction for neuroticism. We also ran $t$ tests to examine whether the differences between the correlations for extraversion and neuroticism were significant. As can be seen in Table 2, the results of these analyses did not reach significance for the word-completion task.

\section{REACTION TIME}

Overall performance. Because extremely slow or extremely fast reaction times could represent responding to something other than the stimulus presented on the screen (e.g., anticipating or guessing the next stimulus, being distracted, or not paying attention on a particular trial), trials on which participants responded in under $200 \mathrm{~ms}$ or over $1,200 \mathrm{~ms}$ were not included in the analyses. As discussed by Fazio (1990), by eliminating these outliers, we were more likely to include only those trials that represent true valence decision times. In analyzing reaction time, we also excluded trials on which participants made errors (pressed the wrong key). Each participant's reaction times were then averaged separately across all of the positive, negative, and neutral trials.

As stated previously, half of the reaction-time trials used verbal stimuli (words), and half used nonverbal stimuli (faces). To assess differences in overall reaction times, we first computed a repeated measures ANOVA, with type of stimulus (verbal or nonverbal) and valence of stimulus (positive, negative, or neutral) as withinsubjects factors. The main effect for type of stimulus was significant, indicating that participants were faster to respond on the nonverbal trials $(M=563.06, S D=58.65)$ than on the verbal trials $(M=705.42, S D=70.57), F(1$, $121)=950.83, p<.001$. The main effect for valence of stimulus was also significant, $F(1,121)=14.02, p<.001$, but the Type $\times$ Valence of Stimulus interaction was not, $F(2,120)=1.99$, ns.

Because performance on the nonverbal trials was faster than performance on the verbal trials, we analyzed the results for these trials separately. The means presented in the middle portion of Table 1 reflect the participants' average reaction times for positive, negative, and neutral trials separately for the verbal and nonverbal trials. Repeated measures ANOVAs on the verbal and nonverbal trials indicated that there were significant differences among the groups in both casesverbal: $F(1,121)=9.41, p<.001$; nonverbal: $F(1,121)=$ $12.96, p<.001$. On both verbal and nonverbal trials, participants were slower to respond to negative stimuli than to neutral stimuli-verbal: $t(121)=-4.00, p<.001$; nonverbal: $t(121)=-5.11, p<.001-$ and positive stimuli-verbal: $t(121)=-2.97, p<.01$; nonverbal: $t(121)=$ $-2.12, p<.10$. For the nonverbal trials, participants were slower to respond to positive stimuli than to neutral stimuli, $t(121)=2.70, p<.03$, but for the verbal trials, the difference between reaction times for positive and neutral stimuli was not significant, $t(121)=0.60, n s$. Overall, participants were more quickly able to identify a stimulus as neutral than as emotionally valenced (positive or negative).

Personality and differential performance. To examine whether personality differentially relates to performance on positive, negative, and neutral emotional stimuli, we computed correlations between extraversion and neuroticism and reaction times for positive, negative, and neutral items for both the verbal and the nonverbal trials. If extraverts are sensitive to positive emotional information, then extraversion should be negatively correlated with reaction time on positive, but not negative or neutral, items. Likewise, if neurotics are sensitive to negative emotional information, then neuroticism should be negatively correlated with reaction time on negative, but not positive or neutral, items. The results of these analyses are presented in the middle portion of Table 2. Supporting our prediction, extraversion was significantly correlated with reaction time to positive verbal stimuli but not with reaction time to negative and neutral verbal items. The relationships between extra- 
version and reaction time for nonverbal stimuli, however, were all nonsignificant. Our prediction for neuroticism was not supported; none of the correlations between neuroticism and reaction-time performance reached significance.

\section{REACTION-TIME ERRORS}

Overall performance. In addition to analyzing reaction time, we also analyzed the number of errors individuals made in response to positive, negative, and neutral stimuli. As stated previously, a response was counted as an error if the participant pressed the wrong key when responding to the stimulus. To assess overall differences in number of errors, we first computed a repeated measures ANOVA, with type of stimulus (verbal or nonverbal) and valence of stimulus (positive, negative, or neutral) as within-subjects factors. The main effect for type of stimulus was significant, indicating that participants made fewer errors on the nonverbal trials $(M=$ 2.13, $S D=3.45)$ than on the verbal trials $(M=3.70, S D=$ 2.84), $F(1,121)=27.92, p<.001$. The main effect for valence of stimulus was not significant, $F(1,121)=0.38$, $n s$, nor was the Type $\times$ Valence of Stimulus interaction, $F(2,120)=1.14$, ns.

The means presented in the middle of Table 1 reflect the average number of errors for positive, negative, and neutral trials separately for the verbal and nonverbal trials. Repeated measures ANOVAs on the verbal and nonverbal trials indicated that there were no significant differences among the groups-verbal: $F(1,121)=0.72$, $n s$, nonverbal: $F(1,121)=2.07$, ns. In general, participants did not make more errors when responding to any particular type of stimulus.

Personality and differential performance. To examine whether personality differentially relates to performance on positive, negative, and neutral emotional stimuli, we computed correlations between extraversion and neuroticism and the number of errors made on positive, negative, and neutral items for both the verbal and the nonverbal trials. The results of these analyses are presented in the middle of Table 2 . On both verbal and nonverbal items, extraversion was significantly correlated with fewer errors on positive stimuli but not neutral or negative stimuli. On the verbal items, neuroticism was significantly correlated with fewer errors on negative stimuli but not neutral or positive stimuli. In other words, extraverts were more accurate (they made fewer errors) in identifying positive items, whereas neurotics were more accurate (they made fewer errors) in identifying negative verbal stimuli. The correlations between neuroticism and performance on the nonverbal stimuli did not reach significance.

\section{FREE RECALL}

Overall performance. The overall means for the total number of positive, negative, and neutral words recalled are presented in the bottom portion of Table 1. A repeated measures ANOVA indicated that there were differences among the means, $F(1,121)=14.52, p<.001$. Participants recalled more positive words than negative words, $t(121)=3.70, p<.001$, and neutral words, $t(121)=$ $4.87, p<.001$. They recalled relatively equal numbers of negative and neutral words, $t(121)=1.79, n s$.

Personality and differential performance. To examine relationships between personality and differential performance on this task, we computed correlations between extraversion and neuroticism and the number of positive, negative, and neutral words recalled (see bottom of Table 2). The results of these analyses supported our predictions for extraversion but not for neuroticism. Extraversion was significantly correlated with the number of positive words recalled but not to the number of negative or neutral words recalled. In other words, extraverts were especially likely to remember positive emotional words but not negative or neutral words. The correlations between neuroticism and recall, however, did not reach statistical significance.

\section{Discussion}

Although the findings were mixed, Study 1 provides some evidence for the differential processing of affective information by extraverts and neurotics. Extraversion was related to faster reaction times for positive verbal stimuli, fewer errors on positive reaction-time items, and better recall of positive words. The results for neuroticism were less supportive of our predictions. Neuroticism was related only to the completion of word fragments as negative and fewer errors on negative reaction-time items. Although the results are mixed, due to the divergent methods used in this study, the fact that any of the predicted correlations emerged as significant is encouraging. Because the effects of personality on the processing of emotional stimuli obtained in this study were rather small, it would be advantageous to replicate these findings before drawing conclusions.

The results for extraversion are consistent with an associative network model interpretation. Extraverts could be characterized as having elaborate associations in memory for positive emotional material, which, in turn, lead to faster valence decisions and better memory for positive stimuli. These findings are consistent with previous research demonstrating that extraversion relates to better retrieval of pleasant memories (Mayo, 1983, 1989). The results for neuroticism were less supportive of this model and were less consistent with prior 
research demonstrating that neuroticism relates to better memory for unpleasant emotional stimuli (Bradley \& Mogg, 1994; Bradley et al., 1993; Desrosiers \& Robinson, 1992; Lishman, 1974; Martin et al., 1983; Mayo, 1983; Okun et al., 1987; Young \& Martin, 1981).

One alternative explanation to the network model is that the relationships between personality and cognitive processing are mediated by current emotional state. As suggested by previous research, extraverts are more likely to experience greater positive emotion, and neurotics are more likely to experience greater negative emotion (Costa \& McCrae, 1980; Emmons \& Diener, 1985; Larsen \& Ketelaar, 1989, 1991; Meyer \& Shack, 1989; O’Malley \& Gillette, 1984; Rusting \& Larsen, 1995; Watson \& Clark, 1992). A large number of studies have demonstrated that such momentary emotional states also facilitate the processing of mood-congruent information (for reviews, see Blaney, 1986; Bower, 1981, 1987; Singer \& Salovey, 1988). When people are in positive moods, they tend to better remember pleasant emotional material; when in negative moods, they tend to better remember unpleasant emotional material. Thus, the findings obtained in Study 1 could have been due to momentary positive and negative emotional states, rather than due directly to the enhanced sensitivity of extraverts and neurotics to positive and negative affective information.

In Study 2, we addressed this alternative explanation. Prior to completing any of the cognitive tasks, participants completed measures of their current mood state. By including measures of baseline positive and negative mood in the analyses, we were able to observe whether personality still relates to cognitive performance when current mood state is taken into account. Study 2 also provided an opportunity to replicate the small effects of personality on cognitive processing obtained in Study 1.

\section{STUDY 2}

\section{Method}

Participants. A total of 108 right-handed undergraduate students ( 70 women, 37 men) participated in the study. Participants were recruited from large upper division psychology courses, and each individual was paid $\$ 10$ for participating.

Procedure. The study was run in two separate sessions, approximately 1 to 2 weeks apart. In Session 1, participants were run in groups of 10 to 30 . They filled out a number of personality measures, including the Extraversion and Neuroticism scales of the EPQ (Eysenck \& Eysenck, 1972). For Session 2, participants were run individually, in approximately 50 -min sessions. During this session, participants completed the same cognitive tasks as in Study 1.

The procedure was exactly the same as Study 1, except we added a measure of current mood state prior to the cognitive tasks. Upon arrival at the laboratory, the experimenter greeted each participant and introduced the experiment with the same cover story as in Study 1. Participants were then asked to fill out the Positive and Negative Affect Schedule (PANAS) (Watson, Clark, \& Tellegen, 1988) as a measure of their current mood state. They were instructed to rate the items according to how they felt "right now" on a scale from 1 (very slightly or not at all) to 5 (extremely). They were told that the purpose of this measure was to make sure that their current feeling state did not influence their performance on the cognitive tasks.

Following the mood measure, each participant completed the same cognitive tasks as in Study 1. The wordcompletion task consisted of the 32 ambiguous stimuli that were used in Study 1. As before, half of the items could be completed as either positive or neutral, and half of the items could be completed as either negative or neutral.

The reaction-time task followed the same procedure and used the same stimuli as in Study 1. Each participant was instructed to decide whether the stimulus was positive, negative, or neutral and to press the corresponding left-, right-, or up-arrow key with his or her right-hand index finger (as before, positive, negative, and neutral overlays were placed over the keys). Participants were told to respond as quickly as possible but to avoid making errors by pressing the wrong key or responding before the stimulus appeared on the screen. They first completed two blocks of 20 practice trials each. Then, participants completed four blocks of trials (two blocks of words; two blocks of faces), each consisting of the same 60 items ( 20 positive, 20 negative, and 20 neutral) as in Study 1. As before, half of the participants received the word blocks first, and half received the face blocks first. The order of presentation of the items in each block was randomized prior to each run.

The recall task also followed the same procedure as in Study 1. Participants were seated at an adjacent desk and asked to recall as many words as they could from the reaction-time task. They were then given $4 \mathrm{~min}$ to recall and record the words onto a blank sheet of paper.

Following the cognitive tasks, each participant was debriefed and thanked for participating. As part of the debriefing procedure, we asked participants to guess our hypotheses. None of the participants were able to guess our specific predictions regarding personality and differential performance on positive and negative stimuli. 
TABLE 3: Descriptive Statistics for Cognitive Tasks, Study 2

\begin{tabular}{lcc}
\hline & $\mathrm{M}$ & $\mathrm{SD}$ \\
\hline Word-fragment completion & & \\
$\quad$ Positive words & 11.15 & 1.94 \\
$\quad$ Negative words & 10.20 & 2.79 \\
Verbal reaction time & & \\
$\quad$ Positive words & 729.73 & 87.53 \\
Negative words & 758.81 & 84.91 \\
$\quad$ Neutral words & 734.55 & 83.01 \\
Nonverbal reaction time & & \\
Positive faces & 615.17 & 103.28 \\
Negative faces & 656.55 & 112.61 \\
$\quad$ Neutral faces & 620.70 & 97.82 \\
Verbal reaction-time errors & & \\
Positive words & 1.58 & 2.51 \\
Negative words & 1.35 & 1.89 \\
Neutral words & 2.59 & 4.85 \\
Nonverbal reaction-time errors & & \\
Positive faces & 0.63 & 0.98 \\
Negative faces & 0.77 & 1.27 \\
Neutral faces & 2.73 & 7.36 \\
Free recall & & \\
Positive words & 5.91 & 2.36 \\
Negative words & 5.64 & 2.33 \\
Neutral words & 5.54 & 1.83 \\
\hline
\end{tabular}

Results

\section{RELATIONSHIPS BETWEEN PERSONALITY AND MOOD}

Correlations between personality and current mood were consistent with prior research investigating relationships between extraversion and neuroticism and positive and negative affect. Extraversion was significantly correlated with positive mood $(r=.23, p<.05)$ but not with negative mood $(r=-.12, n s)$. Neuroticism was significantly correlated with negative $\operatorname{mood}(r=.27, p<.01)$ but not with positive mood $(r=-.16, n s)$. Thus, upon entering the laboratory, those high in extraversion were in a more positive mood than those low in extraversion, and those high in neuroticism were in a more negative mood than those low in neuroticism. However, it should also be noted that these correlations are rather low; the relationships between personality and mood obtained in the current study are not as strong as those found in prior research.

\section{WORD-FRAGMENT COMPLETION}

Overall performance. Means and standard deviations for the number of items participants completed as positive or negative on the word-fragment completion task are presented in the top portion of Table 3. Participants completed more positive word fragments than negative word fragments, $F(1,107)=10.15, p<.01$.

Personality and differential performance. Correlations between extraversion and neuroticism and performance
TABLE 4: Correlations Between Personality and Performance on Cognitive Tasks, Study 2

\begin{tabular}{|c|c|c|c|}
\hline & $\begin{array}{c}\text { Extraversion } \\
\mathrm{r}\end{array}$ & $\begin{array}{c}\text { Neuroticism } \\
\mathbf{r}\end{array}$ & $\begin{array}{c}\text { Difference } \\
\mathbf{t}\end{array}$ \\
\hline \multicolumn{4}{|c|}{ Word-fragment completion } \\
\hline Positive words & $.19 *$ & -.06 & $2.48^{*}$ \\
\hline Negative words & .09 & $.22 *$ & -1.30 \\
\hline \multicolumn{4}{|c|}{ Verbal reaction time } \\
\hline Positive words & $-.22 *$ & -.08 & $-3.01 * *$ \\
\hline Negative words & -.01 & -.18 & 1.68 \\
\hline Neutral words & .04 & .03 & 0.10 \\
\hline \multicolumn{4}{|c|}{ Nonverbal reaction time } \\
\hline Positive faces & $-.19 *$ & .09 & $-2.78 * *$ \\
\hline Negative faces & .15 & -.15 & $2.98 * *$ \\
\hline Neutral faces & .07 & .05 & 0.20 \\
\hline \multicolumn{4}{|c|}{ Verbal reaction-time errors } \\
\hline Positive words & $-.24 *$ & .06 & $-3.01 * *$ \\
\hline Negative words & .06 & $-.19 *$ & $2.48^{*}$ \\
\hline Neutral words & .02 & .15 & -1.28 \\
\hline \multicolumn{4}{|c|}{ Nonverbal reaction-time errors } \\
\hline Positive faces & $-.19 *$ & -.08 & -1.10 \\
\hline Negative faces & .07 & $-.19 *$ & $2.58^{*}$ \\
\hline Neutral faces & .14 & .16 & -0.20 \\
\hline \multicolumn{4}{|l|}{ Free recall } \\
\hline Positive words & $.20^{*}$ & -.11 & $3.09 * *$ \\
\hline Negative words & -.09 & $.21 *$ & $-2.99 * *$ \\
\hline Neutral words & -.03 & .05 & -0.78 \\
\hline
\end{tabular}

$* p<.05 . * * p<.01$.

on the word-fragment trials are presented in the top portion of Table $4 .{ }^{3}$ The results for neuroticism replicated the findings from Study 1; neuroticism was significantly correlated with the number of negative words completed and not with the number of positive words completed. Unlike Study 1, however, extraversion was also significantly correlated with the number of positive words completed and not with the number of negative words completed. In Study 2, therefore, the predictions for both extraversion and neuroticism were supported. Extraverts completed more words as positive, and neurotics completed more words as negative.

Personality, current mood state, and performance. To examine the role of current mood state in performance on the word-completion task, we conducted four separate multiple regression analyses predicting performance on positive and negative words from extraversion and positive mood and from neuroticism and negative mood. By entering personality and mood variables simultaneously, we can observe the effects of personality on performance, while taking current mood state into account. The standardized betas from these analyses are presented in Table 5. Extraversion was still significantly related to the number of positive words completed, and neuroticism was still significantly related to the number of negative words completed. The relationships between 
TABLE 5: Standardized Betas From Multiple Regression Equations Predicting Performance on Cognitive Tasks From Personality and Mood, Study 2

\begin{tabular}{|c|c|c|c|c|}
\hline & & Positive & & Negative \\
\hline & Extraversion & Mood & Neuroticism & Mood \\
\hline Word-fragment co & & & & \\
\hline Positive words & $.19^{*}$ & -.03 & -.02 & -.05 \\
\hline Negative words & -.08 & -.05 & $.21 *$ & .06 \\
\hline Verbal reaction tin & & & & \\
\hline Positive words & $-.23 *$ & .06 & -.12 & -.11 \\
\hline Negative words & -.01 & .00 & -.17 & -.15 \\
\hline Neutral words & .04 & -.03 & .03 & .01 \\
\hline Nonverbal reactio & & & & \\
\hline Positive faces & $-.19 *$ & .03 & .05 & .14 \\
\hline Negative faces & .15 & -.01 & -.18 & -.18 \\
\hline Neutral faces & .09 & -.10 & .00 & .14 \\
\hline Verbal reaction-tin & & & & \\
\hline Positive words & $-.23 *$ & -.03 & .02 & .10 \\
\hline Negative words & .08 & -.09 & $-.19 *$ & -.18 \\
\hline Neutral words & .04 & -.07 & .15 & .01 \\
\hline Nonverbal reactio & rors & & & \\
\hline Positive faces & $-.19 *$ & -.11 & -.06 & -.06 \\
\hline Negative faces & .12 & .04 & -.18 & .03 \\
\hline Neutral faces & .13 & .04 & .14 & .06 \\
\hline Free recall & & & & \\
\hline Positive words & $.19 *$ & .10 & -.06 & -.15 \\
\hline Negative words & -.06 & -.10 & $.19 *$ & .12 \\
\hline Neutral words & -.05 & .05 & .07 & -.05 \\
\hline
\end{tabular}

current mood state and performance, however, did not reach significance.

\section{REACTION TIME}

Overall performance. As in Study 1, we first eliminated extremely slow reaction times (under $200 \mathrm{~ms}$ ) and extremely fast reaction times (over 1,200 ms) from the analyses. We also excluded trials on which participants made errors (pressed the wrong key). Each participant's reaction times were then averaged separately across the positive, negative, and neutral trials.

To assess differences in overall reaction times, we first computed a repeated measures ANOVA, with type of stimulus (verbal or nonverbal) and valence of stimulus (positive, negative, or neutral) as within-subjects factors. As in Study 1, the main effect for type of stimulus was significant, indicating that participants were faster to respond on the nonverbal trials $(M=630.81, S D=93.13)$ than on the verbal trials $(M=741.03, S D=70.78), F(1$, $107)=239.02, p<.001$. The main effect for valence of stimulus was also significant, $F(1,107)=15.16, p<.001$, but the Type $\times$ Valence of Stimulus interaction was not, $F(2,106)=1.55, n s$.

Because performance on the nonverbal trials was faster than performance on the verbal trials, we analyzed the results for these trials separately. The means presented in the middle portion of Table 3 reflect the participants' average reaction times for positive, negative, and neutral trials separately for the verbal and nonverbal trials. Repeated measures ANOVAs on the verbal and nonverbal trials indicated that there were significant differences among the groups in both casesverbal: $F(1,107)=7.79, p<.01 ;$ nonverbal: $F(1,107)=$ $15.80, p<.001$. In both cases, participants were slower to respond to negative stimuli than to neutral stimuliverbal: $t(107)=3.14, p<.01$; nonverbal: $t(107)=4.71$, $p<.001$-and to positive stimuli-verbal: $t(107)=3.67$, $p<.001$; nonverbal: $t(107)=4.73, p<.10$. The differences in reaction times for positive and neutral stimuli were not significant-verbal: $t(107)=0.60, n s$, nonverbal: $t(107)=0.73, n s$.

Personality and differential performance. As in Study 1, we computed correlations between extraversion and neuroticism and reaction times for positive, negative, and neutral items for both the verbal and the nonverbal trials. The results of these analyses are presented in the middle of Table 4. Replicating the results of Study 1, extraversion was negatively correlated with reaction time to positive stimuli but not with reaction time to negative and neutral items. These relationships emerged for both verbal and nonverbal items. Extraverts were particularly fast in identifying positive words and faces. As in Study 1, none of the correlations between neuroticism and reaction time performance reached significance.

Personality, current mood state, and performance. To examine the role of current mood state in performance on the reaction-time task, we conducted six separate multiple regression analyses predicting performance on positive, negative, and neutral verbal stimuli from extraversion and positive mood and from neuroticism and negative mood. We repeated these analyses for the nonverbal stimuli. The standardized betas from these analyses are presented in the bottom section of Table 5 . The relationships between extraversion and reaction time for positive items were still significant for both verbal and nonverbal stimuli. Current mood state, however, was not significantly related to performance on the reaction-time task.

\section{REACTION-TIME ERRORS}

Overall performance. As before, we assessed overall differences in number of errors on the reaction-time task with a repeated measures ANOVA with type of stimulus (verbal or nonverbal) and valence of stimulus (positive, negative, or neutral) as within-subjects factors. The main effect for type of stimulus was significant, indicating that participants made fewer errors on the nonverbal trials $(M=1.38, S D=2.48)$ than on the verbal trials $(M=1.84$, $S D=2.00), F(1,107)=4.04, p<.05$. The main effect for valence of stimulus was also significant, $F(1,107)=8.42$, 
$p<.001$, but the Type $\times$ Valence of Stimulus interaction was not, $F(2,106)=1.97$, $n$.

The means presented in the middle of Table 3 reflect the average number of errors for positive, negative, and neutral trials separately for the verbal and nonverbal trials. Repeated measures ANOVAs on the verbal and nonverbal trials indicated that there were differences in performance on positive, negative, and neutral itemsverbal: $F(1,107)=4.41, p<.05$; nonverbal: $F(1,107)=$ $7.80, p<.01$. On both verbal and nonverbal trials, participants made more errors when responding to neutral items than negative items-verbal: $t(107)=-2.51, p<.05$; nonverbal: $t(107)=-2.72, p<.01-$ or positive itemsverbal: $t(107)=1.95, p<.05$; nonverbal: $t(107)=2.90$, $p<.01$. The differences between errors made in response to positive and negative items were not significantverbal: $t(107)=-0.81, n s$, nonverbal: $t(107)=1.09, n s$.

Personality and differential performance. As in Study 1, we computed correlations between extraversion and neuroticism and the number of errors made on positive, negative, and neutral items for both the verbal and the nonverbal trials. The results of these analyses are presented in the middle of Table 4 . For both verbal and nonverbal items, the findings replicated the results of Study 1. Extraversion was significantly correlated with errors on positive stimuli but not on neutral or negative stimuli; neuroticism was significantly correlated with errors on negative stimuli but not neutral or positive stimuli.

Personality, current mood state, and performance. As before, we conducted six separate multiple regression analyses predicting the number of errors on positive, negative, and neutral verbal stimuli from extraversion and positive mood and from neuroticism and negative mood, and we repeated these analyses for the nonverbal stimuli. The standardized betas from these analyses are presented in the middle of Table 5. The relationships between extraversion and number of errors on positive items were still significant for both verbal and nonverbal stimuli. The relationship between neuroticism and number of errors on negative items was still significant, but only for the verbal stimuli. Once again, the relationships between current mood state and performance did not reach significance.

FREE RECALL

Overall performance. The overall means for the total number of positive, negative, and neutral words recalled are presented in the bottom portion of Table 3. There were no differences in overall recall of positive, negative, or neutral stimuli, $F(1,107)=1.12$, ns.

Personality and differential performance. As in Study 1, we computed correlations between extraversion and neuroticism and the number of positive, negative, and neutral words recalled (see bottom of Table 4). Replicating the results of Study 1, extraversion was significantly correlated with recall of positive words but not neutral or negative words. In addition, neuroticism was significantly correlated with recall of negative words but not neutral or positive words. Extraverts remembered more positive words, whereas neurotics remembered more negative words.

Personality, current mood state, and performance. We conducted the same six separate multiple regression analyses predicting recall of positive, negative, and neutral words from extraversion and positive mood and from neuroticism and negative mood. The standardized betas from these analyses are presented in the bottom of Table 5 . Extraversion was still significantly related to recall of positive words, and neuroticism was still significantly related to recall of negative words. Current mood state was not significantly related to the recall of positive, negative, or neutral words.

\section{Discussion}

The overall pattern of results for Study 2 yielded stronger support for our predictions than Study 1. Extraversion was related to the completion of word fragments as positive, faster reaction times to positive verbal and nonverbal stimuli, fewer errors on positive reaction-time items, and better recall of positive words. Neuroticism was related to the completion of word fragments as negative, fewer errors on negative reaction-time items, and better recall of negative words. Neuroticism did not, however, relate to faster reaction times to negative words.

Study 2 also demonstrated that the effects of personality on the processing of affective stimuli occur independent of momentary mood state. On almost every task, the relationships between personality and performance were still significant when current mood state was taken into account. These results provide support for the notion that there is a direct link between extraversion and neuroticism and differential sensitivity to positive and negative emotional stimuli, at least when natural mood state is measured. They are also consistent with an associative network model interpretation, in which the personality traits of extraversion and neuroticism may be characterized by elaborate positive and negative associations in memory.

\section{GENERAL DISCUSSION}

The present research investigated the hypothesis that extraverts and neurotics differentially process hedonic information. In previous research, Larsen and Ketelaar $(1989,1991)$ demonstrated that extraverts and neurotics were differentially susceptible to positive and negative mood inductions. These results were interpreted within 
Gray's (1981) neurobiological theory of personality, which suggests that extraversion and neuroticism can be thought of as rooted in biologically based systems that directly promote differential responsivity to positive (reward) and negative (punishment) stimuli. The current series of studies demonstrated that there is also some differential cognitive processing of hedonic stimuli associated with personality. Participants participated in a series of cognitive performance tasks: a word-fragment completion task, a reaction-time task using both verbal and nonverbal stimuli, and a recall task in which participants were instructed to remember as many words as they could from the previous reaction-time task. It was predicted that extraverts would perform better than introverts on these tasks when the stimuli were positive and that individuals high in neuroticism would perform better than stable individuals on these tasks when the stimuli were negative.

Although statistical analyses generated some mixed results, our predictions received some support across Studies 1 and 2. Our predictions for extraversion were generally supported in both studies. Extraverts showed better performance on the cognitive tasks when stimuli were positively valenced. Although our predictions for neuroticism received little support in Study 1, they received stronger support in Study 2. In Study 2, neurotics showed better performance on the cognitive tasks (except reaction time) when stimuli were negatively valenced. In addition, Study 2 demonstrated that these personality-cognition relationships are not due to current mood state. There was very little evidence in this study indicating that mood mediates the relationship between personality and cognition.

These findings also add a cognitive perspective to the concepts in Gray's (1981) theory of personality. According to this theory, extraversion and neuroticism arise from individual differences in neurological structures that promote sensitivity to reward and punishment, respectively. Extraverts are thought to be sensitive to rewarding (positive emotional) cues, whereas neurotics are thought to be sensitive to punishing (negative emotional) cues. These predispositions could also be accompanied by a cognitive elaboration of such emotional material in memory, making these positive and negative emotional concepts more readily available and easily retrieved. In addition, because the relations between extraversion and neuroticism and such processing held up after current mood effects were taken into account, we can speculate that the neurological structures proposed by Gray may be represented in memory by a relatively stable cognitive structure. By incorporating the cognitive mechanisms responsible for sensitivity to positive and negative emotional cues into Gray's theory, we may begin to better understand the links between personality and affect found in prior research.

The results are also consistent with previous research demonstrating that extraversion relates to better memory for pleasant stimuli and that neuroticism relates to better memory for unpleasant stimuli (Bradley \& Mogg, 1994; Bradley et al., 1993; Desrosiers \& Robinson, 1992; Lishman, 1974; Martin et al., 1983; Mayo, 1983, 1989; Okun et al., 1987; Young \& Martin, 1981). Study 2 replicated these findings and extended them to include performance in other cognitive domains (e.g., speed of processing) and demonstrated that these personalitycognition relationships are obtained independent of current mood state. These findings suggest a direct relationship between extraversion and neuroticism and sensitivity to positive and negative emotional cues across various cognitive domains.

Before drawing firm conclusions regarding the relationships between personality and cognition, however, it is important to note that the current study used a natural mood measure as a measure of momentary affect. Mood state, as measured in the current study, was, therefore, less intense than if we had induced a strong emotional state, as used in most prior research investigating the impact of mood state on cognitive processing (e.g., Blaney, 1986; Bower, 1981; Singer \& Salovey, 1988). A study in which a stronger emotional state is induced might yield stronger mood-congruency effects than obtained in the current research. However, by simply measuring mood state without inducing an emotional state, we have perhaps more closely approximated individuals' typical experiences. The personality effects obtained in the current research may, therefore, be fairly accurate reflections of personality-mood-cognition relationships as they occur in everyday life.

In both studies, our predictions for extraversion were better supported than our predictions for neuroticism. The weaker results for neuroticism are surprising in light of prior research demonstrating correlations between neuroticism and susceptibility to negative affect that are similar in magnitude to correlations between extraversion and susceptibility to positive affect (Larsen \& Ketelaar, 1989, 1991). One possible explanation for this pattern of findings can be inferred from recent studies showing that neurotics are reactive to all emotional cues (e.g., McFatter, 1994; Wallace, Newman, \& Bachorowski, 1991). For example, Wallace and Newman (1990) found that individuals who were high in both extraversion and neuroticism performed a circle-tracing task fastest in the face of positive rewards, whereas individuals who were high in neuroticism and low in extraversion performed the same task fastest in the face of negative punishments. The authors of this study viewed neuroticism as a "gain" factor that magnifies or dampens the emotional tenden- 
cies due to one's standing on the extraversion-introversion dimension. In other words, according to this approach, neurotic individuals should be sensitive to both positive emotional signals (reward) and negative emotional signals (punishment). If neurotics are sensitive to all emotional cues, then they might also interpret neutral stimuli as emotional. Such sensitivity would obscure any differential effects of neuroticism on performance on positive, negative, and neutral trials and could possibly account for the lack of support for many of our predictions for neuroticism.

Prior research on personality and emotion has also sometimes found evidence for the operation of inhibitory mechanisms in response to positive and negative emotional stimuli. For example, in the Larsen and Ketelaar (1991) study, extraversion was negatively correlated with the intensity of negative mood following the negative mood induction, and neuroticism was negatively correlated with positive mood following the positive mood induction. These findings suggest that extraverts inhibit their reactions to negative emotional stimuli and that neurotics inhibit their reactions to positive stimuli. One of the main differences between the current series of studies and the Larsen and Ketelaar study is that in the Larsen and Ketelaar study, positive and negative emotional states were induced, possibly making participants aware of their current emotional state. In the resent research, however, no emotional states were induced; participants simply performed a series of cognitive tasks. Perhaps these differences suggest that the inhibition of positive and negative emotional states by neurotics and extraverts, respectively, requires an explicit awareness of one's emotional state. Interestingly, a few studies in the mood and memory literature have found evidence for similar inhibitory effects on cognition when negative mood states are induced, and these effects appear to be moderated by personality variables (e.g., Parrott \& Sabini, 1990; Smith \& Petty, 1995). The exploration of possible inhibitory effects on emotional processing, and the delineation of the circumstances in which they are found, should be an interesting area for future research.

Although the present research provides some evidence suggesting that extraverts and neurotics differen- tially process affective information and although these findings are consistent with the notion that extraverts and neurotics are sensitive to positive and negative emotional stimuli, our findings cannot tease apart the causal direction of such effects and their influence on subsequent emotional experience. Do the personality traits of extraversion and neuroticism lead to sensitivity to positive and negative emotional cues, which then enhance susceptibility to positive and negative affect? Or does sensitivity to positive and negative emotional cues lead to the development of extraverted or neurotic personality traits and their accompanied positive and negative affect? The current series of studies cannot determine which of these possible causal mechanisms can account for relationships between personality and emotion.

In addition, the relationships between personality traits, mood states, and cognitive processing of affective information are likely to be much more complex than a simple one-to-one correspondence between personality and affective information processing. Not only do temporarily induced positive and negative emotional states influence cognitive processing (for reviews, see Blaney, 1986; Bower, 1981, 1987; Singer \& Salovey, 1988), but mood-management strategies and the interaction of extraversion and neuroticism with other personality traits and situational factors are also likely to influence the cognitive processing of affective information. Although the current research investigated only some of these factors, it does provide a necessary step toward understanding a possible cognitive mechanism for the links between personality and emotional experience found in prior research.

Future research examining extraversion and neuroticism and cognitive processing of affective information is needed. In addition to exploring the roles of possible inhibitory mechanisms and mood-management strategies, future studies might further delineate the causal mechanisms responsible for relationships among personality, cognition, and emotion. Such research would further our knowledge of these basic personality dimensions and their accompanying biological, cognitive, and experiential components. 


\begin{tabular}{|c|c|c|}
\hline \multicolumn{3}{|c|}{$\begin{array}{l}\text { APPENDIX A } \\
\text { Ambiguous Terms Used in the Word-Fragment } \\
\text { Completion Task and Possible Answers }\end{array}$} \\
\hline Ambiguous Term & Neutral Answer & Positive Answer \\
\hline THR I_- & thrift & thrill \\
\hline $\mathrm{CHE}_{-}$ & chess & cheer \\
\hline PLEA_ED & pleated & pleased \\
\hline _ CITED & recited & excited \\
\hline$\overline{\mathbf{E}}_{-} \mathrm{A}_{-} \mathbf{E} \mathrm{D}$ & erased & elated \\
\hline $\mathrm{CAL}_{-}^{-}$ & calf & calm \\
\hline $\mathrm{GO}_{-} \mathrm{D}$ & gold & good \\
\hline$M E \bar{R} \_Y$ & mercy & merry \\
\hline _- PP $\bar{Y}$ & puppy & happy, peppy \\
\hline$\overline{\mathrm{JO}}_{-}$ & job, jog, jot & joy \\
\hline $\mathrm{DE} \overline{\mathrm{L}}_{-} \mathrm{G}_{-} \mathrm{TED}$ & delegated & delighted \\
\hline$S_{-}$I L I NG & soiling & smiling \\
\hline$\overline{F U}_{-}$ & fur & fun \\
\hline RELA_ED & related & relaxed \\
\hline LI_ELY & likely & lively \\
\hline _L $\bar{E} E$ & flee & glee \\
\hline $\bar{T}$ E N & tenor & tense \\
\hline $\mathrm{ANG}_{--}^{--}$ & angle, angel & anger, angry \\
\hline $\mathrm{MA}_{-}$ & man, may, map, mat & $\operatorname{mad}$ \\
\hline$G L \bar{O}_{--} Y$ & glossy & gloomy \\
\hline _ PRES SED & repressed & depressed \\
\hline$-\bar{U}$ R I O U S & curious & furious \\
\hline$\overline{\mathrm{D}}$ I S_U S & discuss & disgust \\
\hline$A_{-} \bar{O} Y$ & alloy & annoy \\
\hline $\mathrm{F} \mathrm{R}_{-} \mathrm{T}$ & frat & fret \\
\hline $\mathrm{AF}_{-}^{-} \mathrm{AI}_{-}$ & affair & afraid \\
\hline _ O R R O W & borrow & sorrow \\
\hline$\overline{\mathrm{I}} \mathrm{R}$ RI_A TE & irrigate & irritate \\
\hline $\mathrm{G}_{-} \mathrm{I}_{-} \overline{\mathrm{T} Y}$ & gritty & guilty \\
\hline$P \bar{A}_{-}-\bar{N} E D$ & panned & pained \\
\hline $\mathrm{AS}_{-}^{-} \mathrm{MED}$ & assumed & ashamed \\
\hline $\mathrm{HO}_{-} \mathrm{ELESS}$ & homeless & hopeless \\
\hline
\end{tabular}

APPENDIX B

Items Used in the Verbal Reaction-Time Task

\begin{tabular}{lll}
\hline Positive & Negative & Neutral \\
\hline cheerful & unhappy & envelope \\
carefree & hopeless & shoelace \\
delighted & miserable & telephone \\
enthusiastic & sluggish & calendar \\
excited & gloomy & skillet \\
glad & sad & table \\
lively & guilty & store \\
elated & ashamed & hexagon \\
happy & dreary & house \\
pleased & annoyed & middle \\
peppy & fearful & truck \\
affectionate & distressed & fingernail \\
relaxed & nervous & center \\
calm & lonely & street \\
joyful & grouchy & fastener \\
peaceful & pained & cabinet \\
merry & afraid & circle \\
ecstatic & worrying & clipboard \\
overjoyed & sorrowful & driveway \\
thrilled & depressed & light bulb \\
\hline
\end{tabular}

\section{NOTES}

1. Most cognitive tasks in the emotion and cognition literature use verbal stimuli (emotionally valenced words). We included faces to investigate whether personality relates to cognitive processing of nonverbal stimuli and to explore the generality of such emotional processing in relation to extraversion and neuroticism.

2. Correlations were also computed separately for men and women for each of the cognitive tasks in Study 1. For many of the tasks, the differential correlations between personality and performance on emotional stimuli were stronger for women than for men.

3. All analyses were also run separately for men and women for each of the cognitive tasks in Study 2. The gender differences found in Study 2 were similar to those found in Study 1, in which the correlations between personality and performance on emotional stimuli were once again stronger for women than for men. The basic pattern of correlations between personality and performance on positive, negative, and neutral stimuli, however, was similar for men and women.

\section{REFERENCES}

Blaney, P. H. (1986). Affect and memory: A review. Psychological Bulletin, 99, 229-246.

Bower, G. H. (1981). Mood and memory. American Psychologist, 36, 129-148.

Bower, G. H. (1987). Commentary on mood and memory. Behaviour Research and Therapy, 25, 443-456.

Bradley, B. P., \& Mogg, K. (1994). Mood and personality in recall of positive and negative information. Behaviour Research and Therapy, 32, 137-141.

Bradley, B., Mogg, K., Galbraith, M., \& Perrett, A. (1993). Negative recall bias and neuroticism: State vs. trait effects. Behaviour Research and Therapy, 31, 125-127.

Clark, D. M., \& Teasdale, J. D. (1985). Constraints on the effects of mood on memory. Journal of Personality and Social Psychology, 48, 1595-1608.

Costa, P. T., \& McCrae, R. R. (1980). Influence of extraversion and neuroticism on subjective well-being: Happy and unhappy people. Journal of Personality and Social Psychology, 38, 668-678.

Desrosiers, G., \& Robinson, D. (1992). Memory and hedonic tone: "Personality" or "mood" congruence? Psychological Medicine, 22, 117-129.

Emmons, R. A., \& Diener, E. (1985). Personality correlates of subjective well-being. Personality and Social Psychology Bulletin, 11, 89-97.

Eysenck, H.J., \& Eysenck, S.B.G. (1972). Manual of the Eysenck Personality Questionnaire. San Diego, CA: Educational and Industrial Testing Service.

Fazio, R. H. (1990). A practical guide to the use of response latency in social psychological research. Review of Personality and Social Psychology, 11, 74-97.

Gray,J.A. (1981). A critique of Eysenck's theory of personality. In H.J. Eysenck (Ed.), A model for personality (pp. 246-276). New York: Springer.

Larsen, R. J., \& Diener, E. (1992). Promises and problems with the circumplex model of emotion. Review of Personality and Social Psychology, 13, 25-59.

Larsen, R. J., \& Ketelaar, T. (1989). Extraversion, neuroticism, and susceptibility to positive and negative mood induction procedures. Personality and Individual Differences, 10, 1221-1228.

Larsen, R. J., \& Ketelaar, T. (1991). Personality and susceptibility to positive and negative emotional states. Journal of Personality and Social Psychology, 61, 132-140.

Lishman, W. A. (1974). The speed of recall of pleasant and unpleasant experiences. Psychological Medicine, 4, 212-218.

Martin, M., Ward, J. C., \& Clark, D. M. (1983). Neuroticism and the recall of positive and negative personality information. Behaviour Research and Therapy, 21, 495-503.

Mayer,J. D., \& Volanth, A. J. (1985). Cognitive involvement in the mood response system. Motivation and Emotion, 9, 261-275.

Mayo, P. R. (1983). Personality traits and the retrieval of positive and negative memories. Personality and Individual Differences, 4, 465-471. 
Mayo, P. R. (1989). A further study of the personality-congruent recall effect. Personality and Individual Differences, 10, 247-252.

McFatter, R. M. (1994). Interactions in predicting mood from extraversion and neuroticism. Journal of Personality and Social Psychology, $66,570-578$.

Meyer, G. J., \& Shack, J. R. (1989). The structural convergence of mood and personality: Evidence for old and new "directions." Journal of Personality and Social Psychology, 57, 697-706.

Okun, M. A., Stock, W. A., Snead, L., \& Wiermaa, P. (1987). Neuroticism and autobiographical memory for positive and negative events. Personality and Individual Differences, 8, 965-967.

O'Malley, M. N., \& Gillette, C. S. (1984). Exploring the relations between traits and emotions. Journal of Personality, 52, 274-284.

Parrott, W. G., \& Sabini, J. (1990). Mood and memory under natural conditions: Evidence for mood incongruent recall. Joumal of Personality and Social Psychology, 59, 321-336.

Ruiz-Caballero, J. A., \& Gonzalez, P. (1995). Neuroticism, mood, and retrieval of negative personal memories. Journal of General Psychology, $122,29-35$.

Rusting, C. L., \& Larsen, R. J. (1995). Moods as sources of stimulation: Relationships between personality and desired mood states. Personality and Individual Differences, 18, 321-329.

Singer, J. A., \& Salovey, P. (1988). Mood and memory: Evaluating the network theory of affect. Clinical Psychology Review, 8, 211-251.

Smith, S. M., \& Petty, R. E. (1995). Personality moderators of mood congruency effects on cognition: The role of self-esteem and nega- tive mood regulation. Journal of Personality and Social Psychology, 68, 1092-1107.

Teasdale, J. D., \& Russell, M. L. (1983). Differential effects of induced mood on the recall of positive, negative and neutral words. British Journal of Clinical Psychology, 22, 163-171.

Wallace, J. F., \& Newman, J. P. (1990). Differential effects of reward and punishment cues on response speed in anxious and impulsive individuals. Personality and Individual Differences, 11, 999-1009.

Wallace, J. F., Newman, J. P., \& Bachorowski, J. (1991). Failures of response modulation: Impulsive behavior in anxious and impulsive individuals. Journal of Research in Personality, 25, 23-44.

Watson, D., \& Clark, L. A. (1992). On traits and temperament: General and specific factors of emotional experience and their relation to the five-factor model. Journal of Personality, 60, 441-476.

Watson, D., Clark, L. A., \& Tellegen, A. (1988). Development and validation of brief measures of positive and negative affect: The PANAS Scales. Journal of Personality and Social Psychology, 54, 10631070.

Williams, J.M.G., Watts, F. N., McLeod, C., \& Mathews, A. (1988). Cognitive psychology and emotional disorders. Chichester, UK: Wiley.

Young, G.C.D., \& Martin, M. (1981). Processing of information about self by neurotics. British Journal of Clinical Psychology, 20, 205-212.

Received August 8, 1995

Revision accepted August 20, 1997 